get better drainage, I made an opening through the wall of the sinus into the nose. The bleeding was profuse for a few minutes, and with the blood came a quantity of pus. I next trephined the frontal sinus and found it free from pus, but there was a considerable swelling behind the scar mentioned above, and I have no doubt that this was the seat of a subcutaneous fracture where healing had taken place without interference. Both sinuses were thoroughly washed with a gentle stream from a hose pipe, the force of water being sufficient to thoroughly clear the nose of all blood clots and discharges. The wounds were plugged and the horse left for the night.

During the evening about five or six ounces of blood-stained pus came from the wound, and I was not satisfied with the means of drainage provided. Cleaning out the cavity of the wound, the inferior maxillary sinus seemed completely filled with granulation tissue, in the midst of which pus had burrowed in all directions. There had been no further discharge from the nose, so I inferred that the nasal opening had become occluded. I decided to allow it to heal and to trephine below the external wound. The point of the trephine was inserted about an inch anterior to the inferior extremity of the zygomatic process, and the same distance above it. The aperture thus made opened on to the septum, exposing both superior and inferior sinuses, from which three or four ounces of blood-stained pus at once escaped, and a further quantity when the wound was flushed with water. The new tissue in the lower sinus was thoroughly broken down between the original wound and that just made, and all pus carefully washed out, after which all three sinuses were irrigated with carbolic solution and the frontal and maxillary sinus openings plugged with corks, that in the latter having a hole in its centre to allow of the discharge draining freely away. The patient's face was washed, dried, and well coated with corbolised vaseline.

On the $25^{\text {th }}$ the opening into the frontal sinus was allowed to heal, the granulation in the lower wounds removed with the knife, and a gauze drain inserted. Twice every day the wounds were well syringed out with either chinosol or zinc chloride solution. On the 28th the gauze drain was removed and the original wound allowed to heal, only the lower opening to the inferior maxillary sinus being kept open and dressed daily. On the 3 ist all the wounds except the latter were practically healed, and the nasal discharge of no consequence, the horse feeding well and all swelling of the face gone. The lower opening was completely healed on 8th August, and the patient discharged from hospital cured.

\title{
OPERATION ON THE NOSE IN A DOG.
}

By the Samc.

ABOUT a year ago I was consulted about a Retreiver dog, aged about ten years, which was reported to be suffering from an ordinary cold in the nose. In spite of treatment the discharge from the nostrils continued more or less throughout the winter. In January the dog was brought to me with a history of severe intermittent bleeding from the right nostril. I kept him for a week and failed to find any obvious 
cause, so advised his being kept very quiet and carefully dieted. In June he was reported to be much worse again, and in July he came to me once more, and I had the pleasure of showing him at the meeting of the S.C. V. S. on the 7 th. My diagnosis of his case was that of polypus, and I had already made up my mind to make an exploratory opening in the nostril, and also into the frontal sinus in case of there being a pentastome in the latter situation.

On I4th July I trephined the skull and nostril in the following manner. The forehead and nostril on the right side were shaved and thoroughly prepared, and the dog then put under chloroform. When completely anasthetised a probe was used to test the patency of each nostril, and to find if possible the situation of the suspected growth. As I could, however, gain no information from this, I made an incision from the middle line of the nose immediately above the nasal branch of the angular vein, continuing it downwards and backwards below the level of the lower eyelid, and just behind the dental foramen, thus escaping the larger bloodvessels, to meet another incision starting from midway between the inner canthus of the eye and the middle line of the nose. The triangular flap of skin was dissected up and held back, and the bone exposed. All hæmorrhage was at once stopped by use of solution (I-2000) of adrenalin (takamine). The bone was then removed with a half-inch trephine and the nasal cavity exposed. Hæmorrhage, which threatened to be profuse, was once more checked by adrenalin, and the head was kept in a dependent position for fear of fluids entering the larynx by the naso-pharynx, the opening of which was immediately below the operation wound. After careful exploration all I could find was a tumefied condition of the lining membrane, which in two places seemed to resemble small mucous polypi. I removed these, and enlarged the opening a little higher, so as to more fully expose the turbinated bones. Beyond being covered with a very thick mucus, these appeared normal. I introduced a dental mirror into the cavity and examined the walls, but could find no trace of new growths or of any ulceration. The condition of the patient remaining very good, I decided to open the frontal sinus. This was done by making only one incision in the skin about half an inch from the middle line and parallel with it. The lips of the wound were then drawn apart and the bone exposed. The trephine was placed in the centre of this wound, with the centre piece on a line joining the highest points of the two orbital rims. The sinus was found to contain a quantity of mucus but no pentastome; the membrane, however, showed pink spots like small granulations. As the dog had now been under chloroform quite long enough, I syringed out the sinus and nostril, removing all small blood clots, and, as the dog recovered consciousness, tested the patency of the naso-pharynx. This appeared quite free, but the nares seemed more or less occluded and respiration was to a great extent performed through the operation wound. The sinus was cleaned out with sterile swabs and then tlushed out with a I-I 500 solution of perchloride. The skin wound over the frontal sinus was left untouched, but the triangular fiap was stitched with silkworm gut; dried carefully, and sealed with "New Skin."

The patient had been under chloroform for about an hour and twenty minutes, but made an excellent recovery. The administration 
of the chloroform was rather difficult, as the use of an inhaler interfered with the site of the operation, but this was overcome by using a Junker (metal) inhaler, and only just inserting the end of the nose inside the rubber at the end. The preparation "New Skin," which I used to cover the wound, I have found vastly superior to all collodion preparations, as it gives a much thicker covering, dries quicker, and keeps the wound quite aseptic, and animals do not interfere with wounds protected by it.

From this date onward the wounds healed without interruption, and the nose bleeding entirely ceased. There was always a little discharge from the nose, best seen in the morning, but I could find very little interference with normal respiration. The dog was discharged from hospital on 3 Ist July.

\section{A CASE OF HYDRONEPHROSIS IN A COW.}

\section{Bÿ A. SPREUlL, F.R.C.V.S., Dundee.}

On 2 1st June last I was called to see an Irish-bred, eight or rineyears-old, shorthorn-cross, milch cow, the property of a dairy farmer in this neighbourhood. On arrival I found my patient looking rather dejected, suffering from profuse foetid diarrhoea, which the owner informed me had been continuous for a considerable time, but the exact date of its existence could not be ascertained. The skin was dirty and uramic looking, more especially along the region of the back and the upper parts of the shoulders, the coat staring, and accompanied by a considerable amount of itching throughout this region. The pulse, breathing, and temperature were practically normal, the salient symptoms of the case being, as already stated, the profuse foetid diarrhoea and the unhealthy itching coat. The appetite, the owner informed me, was very capricious; some days she would eat little or nothing, while other days she would eat anything she was offered, and at such times looked as if there was little or nothing the matter with her. The owner further informed me that he had bought her about three months previously, and that she had continued during the greater part of that time more or less in this condition, but if anything getting rather worse than better, and hence his appeal for my help.

Without pretending to accurately diagnose the case, I proceeded to prescribe for her, gave astringent antiseptics to assist in regulating the state of the bowels, and put her on hay, cotton cake, and crushed grain as food, with a skin dressing of whale oil and spirits of tar, to be followed after a few days with a good wash with warm water in which a sufficient admixture of Jeyes' fluid had been placed, to rid the hide of the dirty, greasy, matted material there accumulated. For a week or two she seemed to improve somewhat, but all of a sudden one day when the owner entered the byre to see her he found her, as he described it, in a dying condition. He there and then promptly had her killed and sent to the inspection department of the public slaughter-house, where she next came under my observation. Here the carcase was found to be well nourished, fairly fat, weighing thirty-four stones after removal of the kidneys, and 